\title{
PRIMITIVITY OF PRIME COUNTABLE-DIMENSIONAL REGULAR ALGEBRAS
}

\author{
PERE ARA AND JASON P. BELL \\ (Communicated by Birge Huisgen-Zimmermann)
}

\begin{abstract}
Let $k$ be a field and let $R$ be a countable-dimensional prime von Neumann regular $k$-algebra. We show that $R$ is primitive, answering a special case of a question of Kaplansky.
\end{abstract}

\section{INTRODUCTION}

A ring $R$ is von Neumann regular if it has the property that for every $a \in R$ there is some $x \in R$ such that $a=a x a$. If we have $a=a x a$, then $a x$ is necessarily idempotent and so one of the interesting consequences of this condition is that principal left and right ideals of $R$ are generated by idempotents. Kaplansky [9, p. 2] asked whether being prime and being primitive are equivalent properties for von Neumann regular rings.

Fisher and Snider [8] showed that Kaplansky's question has an affirmative answer for a von Neumann regular ring $R$ if $R$ is either a countable ring or if $R$ has a countable downward cofinal set $\mathcal{S}$ of nonzero two-sided ideals (meaning that every nonzero two-sided ideal contains an element of $\mathcal{S}$ ). Domanov [7] later constructed a counterexample by giving an example of a von Neumann regular prime-but-notprimitive group algebra. Further examples were later given by using Leavitt path algebra constructions [1].

These counterexamples all have the property that they are uncountably infinite dimensional over their base fields and it is therefore natural to ask whether Kaplansky's question has an affirmative answer if one adds the additional hypothesis that the algebra be countable-dimensional. When the base field is countable this follows from Fisher and Snider's theorem since such algebras are necessarily countable as rings. On the other hand, Fisher and Snider's work says nothing about von Neumann regular algebras over uncountable fields unless one has the countable cofinality condition on the set of two-sided ideals.

In this paper, we answer Kaplansky's question for countable-dimensional rings.

Received by the editors September 11, 2013 and, in revised form, October 15, 2013 and November 18, 2013.

2010 Mathematics Subject Classification. Primary 16E50; Secondary 16D60, 16N60.

Key words and phrases. von Neumann regular rings, primitive rings, prime rings, idempotents, extended centroid, multiplier rings.

The first-named author was partially supported by DGI MINECO MTM2011-28992-C02-01, by FEDER UNAB10-4E-378 "Una manera de hacer Europa", and by the Comissionat per Universitats i Recerca de la Generalitat de Catalunya.

The second-named author was supported by NSERC grant 31-611456. 
Theorem 1.1. Let $k$ be a field and let $R$ be a prime von Neumann regular $k$-algebra with $\operatorname{dim}_{k}(R) \leq \aleph_{0}$. Then $R$ is both left and right primitive.

Indeed, the class of algebras to which our argument applies is a much larger class than the class of prime regular rings, namely the class of prime algebras such that every nonzero right ideal $I$ of $R$ contains a nonzero idempotent $e$ with the property that the center $Z(e R e)$ is a field. Thus we shall show that such algebras are automatically primitive when $R$ is countable-dimensional (Theorem 3.2). The class of algebras $R$ such that every nonzero right ideal of $R$ contains a nonzero idempotent is very large. It contains, amongst others, all exchange semiprimitive algebras [10, all Leavitt path algebras of arbitrary graphs with the property (L), see e.g. [11, Theorem 1], all Kumjian-Pask algebras of aperiodic row-finite higherrank graphs without sources, see [2, Proposition 4.9], and all abelianized Leavitt path algebras $L_{K}^{\mathrm{ab}}(E, C)$ of finite bipartite separated graphs $(E, C)$ with condition (L) [4, Theorem 10.10]. The condition about the centers $Z(e R e)$ of the corner algebras holds in many examples, and is automatic when the prime algebra $R$ is centrally closed, which is the case for prime Leavitt path algebras of finite graphs, by [6. Theorem 3.7].

The outline of the paper is as follows. In Section 2, we give some background on the extended centroid and on multiplier rings. We use this material to show that if $R$ is a countable-dimensional prime von Neumann regular algebra (or satisfies the weaker hypothesis alluded to in the previous paragraph), whose base field is uncountable, then the extended centroid of $R$ is an algebraic extension of the base field. This fact is then used in Section 3 to prove our main result.

\section{The EXTENDED CENTROID AND MULTIPLIER RINGS}

Let $R$ be a semiprime ring (we do not assume that $R$ has identity). The multiplier ring $M(R)$ consists of all tuples $(f, g)$ where $f: R \rightarrow R$ is a left $R$-module homomorphism, $g: R \rightarrow R$ is a right $R$-module homomorphism and they satisfy the balanced condition $f(x) y=x g(y)$ for all $x, y \in R$. Addition in this ring is just given by an ordinary coordinate-wise addition of maps and multiplication is given by the rule

$$
\left(f_{1}, g_{1}\right) \cdot\left(f_{2}, g_{2}\right):=\left(f_{2} \circ f_{1}, g_{1} \circ g_{2}\right) .
$$

It is straightforward to check that with these operations $M(R)$ is a ring with identity given by (id,id). Moreover, $R$ embeds as a subring in $M(R)$ via the rule $r \mapsto$ $\left(\mathcal{R}_{r}, \mathcal{L}_{r}\right)$, where $\mathcal{L}_{r}$ and $\mathcal{R}_{r}$ are respectively left and right multiplication by $r$.

We need the following well-known lemma. We include a proof for the reader's convenience.

Lemma 2.1. Let $e$ be an idempotent of a prime ring $R$. Then $Z(M(R e R)) \cong$ $Z(e R e)$.

Proof. Define $\phi: Z(M(R e R)) \rightarrow Z(e R e)$ by $\phi(x)=e x e$, where we identify $R e R$ with a subring of $M(R e R)$ in the natural way. Observe that $x e \in R e R$ because $x \in$ $M(\operatorname{Re} R)$ and $e \in R e R$, and thus $e x e=e(x e) e \in e R e$. Since $x \in Z(M(R e R))$ we have $x e=e x=e x e \in e R e$. Using this, we see that $\phi$ is a unital ring homomorphism. If $\phi(x)=0$, then $x e=0$ and so $x\left(\sum_{i} r_{i} e s_{i}\right)=\sum_{i} r_{i}(x e) s_{i}=0$ for all $r_{i}, s_{i} \in$ $R$, which in turn implies that $x=0$. This shows that $\phi$ is injective. To show 
surjectivity, take $x \in Z(e R e)$ and define $f_{x}: R e R \rightarrow R e R$ by

$$
f_{x}\left(\sum_{i} r_{i} e s_{i}\right)=\sum_{i} r_{i} x s_{i} .
$$

To show that $f_{x}$ is well defined, suppose that $\sum_{i} r_{i} e s_{i}=0$. Then for all $t \in R$ we have, using that $x \in Z(e R e)$,

$$
\left(\sum_{i} r_{i} x s_{i}\right) t e=\sum_{i} r_{i} x\left(e s_{i} t e\right)=\left(\sum_{i} r_{i} e s_{i}\right)(t e x)=0 .
$$

This shows that $\sum_{i} r_{i} x s_{i}=0$. Thus $f_{x}$ is a well-defined $R$-bimodule homomorphism and thus $\left(f_{x}, f_{x}\right) \in Z(M(I))$. Clearly $\phi\left(\left(f_{x}, f_{x}\right)\right)=x$. This concludes the proof.

The symmetric ring of quotients of a semiprime ring $R$ can be defined as follows. We let $\mathcal{T}$ denote the collection of triples $(f, g, I)$, where $I$ is an essential ideal of $R$ and $f, g: I \rightarrow R$ are maps with $f$ a left $R$-module homomorphism, $g$ a right $R$-module homomorphism and such that $f(x) y=x g(y)$ for all $x, y \in I$. We put an equivalence relation $\sim$ on $\mathcal{T}$ by declaring that $\left(f_{1}, g_{1}, I_{1}\right) \sim\left(f_{2}, g_{2}, I_{2}\right)$ if $f_{1}$ and $f_{2}$ agree on $I_{1} \cap I_{2}$ and $g_{1}$ and $g_{2}$ agree on $I_{1} \cap I_{2}$. It is straightforward to check that this gives an equivalence relation on $\mathcal{T}$ and we let $[(f, g, I)]$ denote the equivalence class of $(f, g, I) \in \mathcal{T}$. We then define the symmetric ring of quotients, $Q_{s}(R)$, of $R$ to be the collection of equivalences classes $[(f, g, I)]$ with addition and multiplication defined by

$$
\left[\left(f_{1}, g_{1}, I_{1}\right)\right]+\left[\left(f_{2}, g_{2}, I_{2}\right)\right]=\left[\left(h_{1}, h_{2}, I_{1} \cap I_{2}\right)\right]
$$

and

$$
\left[\left(f_{1}, g_{1}, I_{1}\right)\right] \cdot\left[\left(f_{2}, g_{2}, I_{2}\right)\right]=\left[\left(k_{1}, k_{2}, I_{2} I_{1}\right)\right]
$$

where $h_{1}$ is the restriction of $f_{1}+f_{2}$ to $I_{1} \cap I_{2} ; h_{2}$ is the restriction of $g_{1}+g_{2}$ to $I_{1} \cap I_{2} ; k_{1}=f_{2} \circ f_{1}$ and $k_{2}=g_{1} \circ g_{2}$ on $I_{2} I_{1}$. Then $Q_{s}(R)$ is a ring with identity given by the class [(id, id, $R)]$. Furthermore, $R$ embeds in $Q_{s}(R)$ as a subring via the rule $r \mapsto\left[\left(\mathcal{R}_{r}, \mathcal{L}_{r}, R\right)\right]$, and we identify $R$ with its image in $Q_{s}(R)$. We define the extended centroid of $R, C(R)$, to be the centre of $Q_{s}(R)$. We note that if $R$ is a prime ring, then the extended centroid of $R$ is a field [5, Corollary 2.1.9].

In the case of a prime ring $R$, the extended centroid has an alternative description as follows (see e.g. [3, page 359]). We let $\mathcal{B}$ denote the collection of pairs $(f, I)$, where $I$ is a nonzero ideal of $R$ and $f: I \rightarrow R$ is an $(R, R)$-bimodule homomorphism. We place an equivalence relation on $\mathcal{B}$ by declaring that $(f, I) \sim(g, J)$ if $f=g$ on $I \cap J$ and we let $[(f, I)]$ denote the equivalence class of $(f, I) \in \mathcal{B}$. Then the set of equivalence classes forms a ring with addition given by $[(f, I)]+[(g, J)]=$ $[(f+g, I \cap J)]$ and $[(f, I)] \cdot[(g, J)]=[(f \circ g, J I)]$.

We note that if $R$ is prime and $a, b \in R$ are nonzero with $a x b=b x a$ for every $x \in R$, then we have a bimodule isomorphism $f:(a) \rightarrow(b)$ given by $\sum x_{i} a y_{i} \mapsto$ $\sum x_{i} b y_{i}$. To check this it is sufficient to check that $\sum x_{i} a y_{i}=0$ if and only if $\sum x_{i} b y_{i}=0$. We check one direction; the other direction follows from symmetry. Suppose that $\sum x_{i} a y_{i}=0$. Then

$$
\left(\sum x_{i} a y_{i}\right) r b=0
$$

for every $r \in R$. Using the fact that $a x b=b x a$ for every $x \in R$ gives

$$
\left(\sum x_{i} b y_{i}\right) r a=0
$$


for every $r \in R$. Since $a$ is nonzero and $R$ is prime, we see that $\sum x_{i} b y_{i}=0$.

Lemma 2.2. Let $k$ be a field and let $R$ be a prime $k$-algebra such that every nonzero ideal of $R$ contains a nonzero idempotent e such that $Z(e R e)$ is a field. If $\operatorname{dim}_{k}(R)<|k|$, then the extended centroid of $R$ is an algebraic extension of $k$.

Proof. We have $C(R)=Z\left(Q_{s}(R)\right)$. Let $\mathcal{F}$ be the family of nonzero ideals $I$ of $R$ of the form $I=R e R$, where $e$ is an idempotent in $R$ such that $Z(e R e)$ is a field. If $J$ is a nonzero ideal of $R$, then by hypothesis there is $I \in \mathcal{F}$ such that $I \subseteq J$. Note that $I=I^{2}$ for all $I \in \mathcal{F}$. Observe also that, if $I \subseteq J$ are idempotent nonzero ideals of a prime ring $T$, then there is a natural embedding $M(J) \rightarrow M(I)$, and this carries central elements to central elements. Hence, given a downward directed system of idempotent nonzero ideals, one gets a directed system of embeddings of their multiplier rings, and a directed system of embeddings of the centres of these.

By the above remarks and the proof of Proposition 2.1.3 of [5], we may write $C(R)$ as

$$
Z\left(\underset{I \in \mathcal{F}}{\lim _{\longrightarrow}} M(I)\right),
$$

where $\mathcal{F}$ is partially ordered by reverse inclusion. Since $Z\left(\lim _{I \in \mathcal{F}} M(I)\right)=$ $\lim _{I \in \mathcal{F}} Z(M(I))$, it is sufficient to show that $Z(M(I))$ is a field which is an algebraic extension of $k$ whenever $I \in \mathcal{F}$, as this will give that $C(R)$ is a directed limit of algebraic field extensions of $k$. Let $I \in \mathcal{F}$. Then, by definition, there is an idempotent $e$ in $I$ such that $I=R e R$ and $Z(e R e)$ is a field. By Lemma 2.1, we have that $Z(M(I))$ is isomorphic as $k$-algebra to $Z(e R e)$, and thus $Z(M(I))$ is a field. Since $\operatorname{dim}_{k}(e R e)<|k|$, we have that $Z(M(I))$ is an algebraic extension of $k$. Indeed, if $t$ is a transcendental element of $Z(M(I))$, then $\left\{(t-\alpha)^{-1}: \alpha \in k\right\}$ is a set of linearly independent elements of $Z(M(I))$, of cardinality $|k|$, which contradicts the fact that $\operatorname{dim}_{k} Z(M(I))=\operatorname{dim}_{k} Z(e R e)<|k|$. As $C(R)$ is a directed limit of algebraic extensions of $k$, we conclude that $C(R)$ is algebraic over $k$.

Corollary 2.3. Let $k$ be a field and let $R$ be a prime regular $k$-algebra. If $\operatorname{dim}_{k}(R)<$ $|k|$, then the extended centroid of $R$ is an algebraic extension of $k$.

Proof. If $e$ is a nonzero idempotent of $R$, then $e R e$ is a nonzero prime regular ring, and so $Z(e R e)$ is a field. Therefore, the result follows from Lemma 2.2

\section{Countable-Dimensional prime Regular algebras}

Lemma 3.1. Let $k$ be a field, let $R$ be a prime $k$-algebra, and let $V$ be a nonzero finite dimensional subspace of $R$. Suppose that the extended centroid of $R$ is an algebraic extension of $k$. Then there exists a nonzero element $r \in R$ such that $r \in I$ for all two-sided ideals $I$ such that $V \cap I \neq(0)$.

Proof. We prove this by induction on the dimension of $V$. If $\operatorname{dim}(V)=1$, there is nothing to prove. We thus assume that the claim holds whenever $\operatorname{dim}(V)<n$ and we consider the case that $\operatorname{dim}(V)=n$ with $n \geq 2$. Let $\left\{r_{1}, \ldots, r_{n}\right\}$ be a basis for $V$ and let $\mathcal{S}$ denote the collection of two-sided ideals in $R$ with the property that $I \cap V \neq(0)$. Then there exist maps $c_{1}, \ldots, c_{n}: \mathcal{S} \rightarrow k$ such that for each $I \in \mathcal{S}$ we have

$$
\sum_{i=1}^{n} c_{i}(I) r_{i} \in I
$$


and such that for each $I \in \mathcal{S}$ there exists some $i=i(I) \in\{1, \ldots, n\}$ such that $c_{i}(I) \neq 0$. We now consider two cases.

Case I. There is some $j$ and some $x \in R$ such that $r_{j} x r_{1} \neq r_{1} x r_{j}$.

We let $T$ denote the set of elements $\left(b_{2}, \ldots, b_{n}\right) \in k^{n-1}$ such that

$$
\sum_{i=2}^{n} b_{i}\left(r_{i} x r_{1}-r_{1} x r_{i}\right)=0
$$

Then $T$ is a proper subspace of $k^{n-1}$, since $r_{j} x r_{1}-r_{1} x r_{j} \neq 0$. We let

$$
\mathcal{S}_{0}=\left\{I \in \mathcal{S}:\left(c_{2}(I), \ldots, c_{n}(I)\right) \in T\right\} .
$$

For $I \in \mathcal{S}$ we have

$$
\left(\sum_{i} c_{i}(I) r_{i}\right) x r_{1}-r_{1} x\left(\sum_{i} c_{i}(I) r_{i}\right) \in I .
$$

We may rewrite this element as

$$
\sum_{i=2}^{n} c_{i}(I)\left(r_{i} x r_{1}-r_{1} x r_{i}\right)
$$

Furthermore, for $I \in \mathcal{S} \backslash \mathcal{S}_{0}$, we have $I \cap W \neq(0)$, where $W$ is the span of $\left\{r_{i} x r_{1}-r_{1} x r_{i}: i=2, \ldots, n\right\}$. Let

$$
W^{\prime}:=\left\{\sum_{i=1}^{n} b_{i} r_{i}:\left(b_{1}, b_{2}, \ldots, b_{n}\right) \in k^{n} \text { and }\left(b_{2}, \ldots, b_{n}\right) \in T\right\} .
$$

It follows that if $I \in \mathcal{S}$, then either $I \cap W \neq(0)$ or $I \cap W^{\prime} \neq(0)$. Notice that $W^{\prime}$ has dimension $1+\operatorname{dim}(T)<n$, since $T$ is a proper subspace of $k^{n-1}$.

Since both $W$ and $W^{\prime}$ are nonzero and have dimension less than $n$, the inductive hypothesis gives that there exist nonzero elements $a, b \in R$ such that either $a$ or $b$ is in $I$ whenever $I \in \mathcal{S}$. Since $R$ is prime, there exists some $y \in R$ such that $a y b \neq 0$. We thus see that $a y b \in I$ whenever $I \cap V \neq(0)$ giving us the claim in this case.

Case II. $r_{1} x r_{j}=r_{j} x r_{1}$ for all $x \in R$ and all $j=2,3, \ldots, n$.

We use the description of $C(R)$ as equivalence classes of bimodule homomorphisms. We let $F$ denote the extension of $k$ generated by the elements $\left[\left(f_{j},\left(r_{j}\right)\right)\right]$ for $j=1, \ldots, n$, where $f_{j}:\left(r_{1}\right) \rightarrow R$ is given by $x r_{1} y \mapsto x r_{j} y$ and extending via linearity. The argument given in Section 2 shows that the maps $f_{j}$ are well defined.

Since the extended centroid of $R$ is algebraic, and $F$ is finitely generated as a field extension of $k, F$ is a finite extension of $k$. We fix a basis $\left\{h_{1}, \ldots, h_{m}\right\}$ for $F$ as a $k$-vector space. For each $i \in\{1, \ldots, m\}$ there is a nonzero element $b_{i}$ of $R$ with the property that $h_{i}$ sends $b_{i}$ into $R$. We pick a nonzero $b \in R$ that is in the intersection of the ideals generated by $b_{1}, \ldots, b_{m}$. Then by construction, $b F \subseteq R$. Notice that for $I \in \mathcal{S}$, we have

$$
\sum_{i} c_{i}(I) r_{i} \in I
$$

We may rewrite this as

$$
\left(\sum_{i} c_{i}(I) f_{j}\right)\left(r_{1}\right) \in I
$$


Equivalently, there is a map $\beta: \mathcal{S} \rightarrow F \backslash\{0\}$ such that $\beta(I) r_{1} \in V \cap I$. Since $\beta(I)^{-1} \in F$, we have that $b \cdot \beta(I)^{-1} \in R$. Furthermore, $R$ is prime and hence there is some nonzero element $t$ such that $b t r_{1} \neq 0$. Then $\left(b \cdot \beta(I)^{-1} t\right)\left(\beta(I) r_{1}\right)=b t r_{1} \in I$ whenever $I \cap V \neq(0)$ giving us the claim in the remaining case.

We note that the conclusion to the statement of Lemma 3.1 need not hold if the extended centroid of $R$ is not an algebraic extension of the base field. As a simple example, let $k$ be an infinite field and take $R=k[x]$ and let $V=k x+k$. Then for each $\alpha \in k$, we have $R(x-\alpha) \cap V \neq(0)$, but the intersection of $R(x-\alpha)$ as $\alpha$ ranges over $k$ is zero.

We now prove our main theorem. In the proof, we make use of a result of Fisher and Snider that guarantees primitivity of a prime ring under the conditions that each nonzero right ideal of the ring has a nonzero idempotent and such that there is a countable downward cofinal set of nonzero ideals. We recall that a set $\mathcal{S}$ of nonzero ideals in a ring $R$ is downward cofinal if each nonzero ideal of $R$ contains an ideal in $\mathcal{S}$.

Theorem 3.2. Let $k$ be a field and let $R$ be a prime $k$-algebra such that each nonzero right ideal of $R$ contains a nonzero idempotent e such that $Z(e R e)$ is a field. Suppose that $\operatorname{dim}_{k}(R) \leq \aleph_{0}$. Then $R$ is both left and right primitive.

Proof. If $k$ is a countable field, then $R$ is a countable ring and hence $R$ is right and left primitive by [8, Theorem 1.1]. Thus it is no loss of generality to assume that $k$ is uncountable. Thus in this case we have that the extended centroid of $R$ is an algebraic extension of $k$ by Lemma 2.2 .

Since $R$ is countably-infinite or finite dimensional, there exists an increasing sequence of finite-dimensional nonzero subspaces

$$
V_{1} \subseteq V_{2} \subseteq \cdots
$$

such that

$$
R=\bigcup_{i} V_{i}
$$

By Lemma 3.1, there exist nonzero elements $a_{i} \in R$ such that $a_{i} \in I$ whenever $I \cap V_{i} \neq(0)$. We let $J_{i}=\left(a_{i}\right)$ denote the two-sided ideal generated by $a_{i}$. Then since every nonzero ideal in $R$ must intersect some $V_{i}$ we see that the collection $\left\{J_{i}: i \geq 1\right\}$ is a countable downward cofinal set of ideals in $R$. Thus by Fisher and Snider [8, Theorem 1.1], we see that $R$ is both left and right primitive.

We can now obtain a proof of Theorem 1.1.

Proof of Theorem 1.1. Recall that $Z(e R e)$ is a field for every nonzero idempotent $e$ of a prime von Neumann regular ring $R$. Hence the proof follows immediately from Theorem 3.2 .

Since finitely generated algebras are necessarily at most countably infinite dimensional over their base fields, we obtain the following result as an immediate corollary of Theorem 3.2 .

Corollary 3.3. Let $k$ be a field and let $R$ be a finitely generated prime $k$-algebra such that each nonzero right ideal of $R$ contains a nonzero idempotent e such that $Z(e R e)$ is a field. Then $R$ is both left and right primitive. 
We close by recording the equivalences between the various hypotheses that have played a role in the proofs of our results.

Proposition 3.4. Let $k$ be an uncountable field and let $R$ be a prime countable dimensional $k$-algebra such that each nonzero (two-sided) ideal of $R$ contains a nonzero idempotent. Then the following conditions are equivalent:

(i) The extended centroid $C(R)$ is an algebraic field extension of $k$.

(ii) $\operatorname{dim}_{k} C(R) \leq \aleph_{0}$.

(iii) For each nonzero idempotent e of $R$ we have that $Z(e R e)$ is a field.

(iv) Each nonzero ideal of $R$ contains a nonzero idempotent e such that $Z(e R e)$ is a field.

Proof. (i) $\Longrightarrow$ (ii). As in the proof of Theorem $3.2, R$ has a countable downward cofinal chain of two-sided ideals $J_{i}$. Since each contains a nonzero idempotent, we can obtain from them a countable chain of idempotent-generated ideals $R e_{i} R$ (with $\left.e_{i}^{2}=e_{i}\right)$, cofinal among all nonzero ideals of $R$. We have that

$$
C(R)=\underset{i}{\lim } Z M\left(R e_{i} R\right) .
$$

Since $Z M\left(R e_{i} R\right) \cong Z\left(e_{i} R e_{i}\right)$ by Lemma 2.1, we get that $\operatorname{dim}_{k}\left(Z M\left(R e_{i} R\right)\right) \leq \aleph_{0}$, and consequently $\operatorname{dim}_{k} C(R) \leq \aleph_{0}$.

(ii) $\Longrightarrow$ (i). This follows from the facts that $C(R)$ is a field extension of $k$ and that $k$ is an uncountable field (cf. the last part of the proof of Lemma 2.2).

(i) $\Longrightarrow$ (iii). Let $e$ be a nonzero idempotent of $R$. The extension $k \subseteq Z(e R e)$ is algebraic, because $Z(e R e) \subseteq C(R)$. Since $Z(e R e)$ is a domain, it follows that it must be a field.

(iii) $\Longrightarrow$ (iv). Obvious.

(iv) $\Longrightarrow$ (i). This follows from Lemma 2.2 .

\section{ACKNOWLEDGMENTS}

We thank Efim Zelmanov, who suggested this question to us during a ring theory meeting in Poland. We also thank the anonymous referee for carefully reading the paper and for providing many helpful suggestions.

\section{REFERENCES}

[1] Gene Abrams, Jason P. Bell, and Kulumani M. Rangaswamy, On prime nonprimitive von Neumann regular algebras, Trans. Amer. Math. Soc. 366 (2014), no. 5, 2375-2392, DOI 10.1090/S0002-9947-2014-05878-9. MR 3165642

[2] Gonzalo Aranda Pino, John Clark, Astrid an Huef, and Iain Raeburn, Kumjian-Pask algebras of higher-rank graphs, Trans. Amer. Math. Soc. 365 (2013), no. 7, 3613-3641, DOI 10.1090/S0002-9947-2013-05717-0. MR3042597

[3] Pere Ara, The extended centroid of $C^{*}$-algebras, Arch. Math. (Basel) 54 (1990), no. 4, 358364, DOI 10.1007/BF01189582. MR1042128 (91d:46072)

[4] Pere Ara and Ruy Exel, Dynamical systems associated to separated graphs, graph algebras, and paradoxical decompositions, Adv. Math. 252 (2014), 748-804, DOI 10.1016/j.aim.2013.11.009. MR.3144248

[5] Pere Ara and Martin Mathieu, Local multipliers of $C^{*}$-algebras, Springer Monographs in Mathematics, Springer-Verlag London Ltd., London, 2003. MR1940428 (2004b:46071)

[6] M. G. Corrales Garcia, D. Martin Barquero, C. Martin Gonzalez, M. Siles Molina and J. F. Solanilla Hernandez, Extreme cycles. The center of a Leavitt path algebra, arXiv:1307.5252v1 [math.RA].

[7] O. I. Domanov, A prime but not primitive regular ring (Russian), Uspehi Mat. Nauk 32 (1977), no. 6(198), 219-220. MR0573043 (58 \#28058) 
[8] Joe W. Fisher and Robert L. Snider, Prime von Neumann regular rings and primitive group algebras, Proc. Amer. Math. Soc. 44 (1974), 244-250. MR0342551 (49 \#7297)

[9] Irving Kaplansky, Algebraic and analytic aspects of operator algebras, American Mathematical Society, Providence, R.I., 1970. Conference Board of the Mathematical Sciences Regional Conference Series in Mathematics, No. 1. MR0312283 (47 \#845)

[10] W. K. Nicholson, Lifting idempotents and exchange rings, Trans. Amer. Math. Soc. 229 (1977), 269-278. MR0439876 (55 \#12757)

[11] Kulumani M. Rangaswamy, Leavitt path algebras which are Zorn rings, Ring theory and its applications, Contemp. Math., vol. 609, Amer. Math. Soc., Providence, RI, 2014, pp. 277-283, DOI 10.1090/conm/609/12119. MR.3204366

Departament de Matemàtiques, Universitat Autònoma de Barcelona, 08193 Bellaterra, Barcelona, Spain

E-mail address: para@mat.uab.cat

Department of Pure Mathematics, University of Waterloo, Waterloo, Canada

E-mail address: jpbell@uwaterloo.ca 